\title{
PROGRAMMABLE PROCESS STRUCTURE BASED ANALYSIS OF HY- DROGEN SUPPLY CHAINS
}

\author{
Mónika Varga *1, Catherine Azzaro-Pantel ${ }^{2}$, José Manuel Flores-Perez ${ }^{2}$, and Béla \\ CSUKÁS ${ }^{1}$
}

\author{
${ }^{1}$ Institute of Methodology, University of Kaposvár, Guba Sándor u. 40, Kaposvár, 7400, HUNGARY \\ ${ }^{2}$ Laboratoire de Génie Chimique, Université de Toulouse, 4 Allée Emile Monso, Toulouse, 31030, FRANCE
}

\begin{abstract}
The planning and operation of Hydrogen Supply Chains (HSC) often require easily extensible, generic dynamic simulation tools. In this paper, the non-conventional modeling and simulation methodology of Programmable Process Structures is applied for the description of these process systems. Programmable Process Structures of HSC models are generated from the two general functional meta-prototypes of the method and from the description of the studied HSC network. The actual program prototypes of production, transformation, transportation, utilization and intermediate storage are copied from the meta-prototypes and filled with the locally executable declarative program code for the various classes of elements. The actual state and transition elements are parameterized and initialized according to their case-specific prototypes. The execution of the programmed HSC structures is solved by the general purpose kernel program. The application of methodology, developed in other fields, is illustrated by a fictitious, simplified HSC example. Analysis of this example model illustrates that the coordination of the hectically changing energy production of renewable resources with its seasonally and tendentiously changing demands is in need of dynamic simulation-based planning.
\end{abstract}

Keywords: Hydrogen Supply Chains, dynamic simulation, model generation, simulation-based planning, Programmable Process Structures

\section{Introduction}

According to the recently published special section of the AIChE Journal about "Hydrogen Deployment" [1], the importance of hydrogen energy is expected to grow in the future because stakeholders have just started to discover the potential advantages of it worldwide [2]. Hydrogen Supply Chains (HSCs) contain geographically distributed, heterogeneous elements, while many of them are time-specifically constrained by meteorological or seasonal characteristics. Having analyzed some comprehensive case studies [3-5], it is obvious that the zero emissions of hydrogen burning and the relatively easy storage of hydrogen is perspectivic. At present, a deadlock exists because a lack of infrastructure inhibits production, while a lack of production impedes the development of infrastructure. Dynamic modeling and simulation-based design tools may play an important role in accelerating developments.

Primary renewable resources of hydrogen production are solar radiation, wind power and hydroelectric power produced by environmental systems. The utilization of solar energy requires natural or cultivated land, which is a special finite resource that is also generated for use in other industries, e.g. food-producing agriculture.

\footnotetext{
*Correspondence: varga.monika@ke.hu
}

The direct utilization of solar energy is achieved by various photovoltaic processes with a limited degree of efficiency and capacity. Intensive research and developments are in progress that make use of many new resources involving special organisms (e.g. microalgae [6]) or artificial biomimetic systems (e.g. artificial leaves [7]).

The indirect utilization of solar energy is realized by the construction of power plants, which also compete for land suitable for food production.

Wind farms and some smaller hydroelectric power stations produce electrical energy periodically (often randomly), so on-site conversion to electrical energy is necessary for their effective utilization. However, electrical grids do not tolerate such erratic changes and no good solutions for temporary electricity buffering and storage exist.

Primary non-renewable resources are nuclear raw materials, natural gas and coal. Nuclear raw materials need to be transported to specifically located nuclear power stations. Natural gas is available from widespread pipelines. On the contrary, coal-based processing requires stations to be constructed in the vicinity of mining sites.

Large hydroelectric and nuclear power plants produce easily transportable electrical energy that can contribute to buffered hydrogen production by on-site electrolysis. 
The most frequently applied process, Steam Methane Reforming (SMR), competes with other applications of natural gas but can produce hydrogen-containing gas anywhere in the vicinity of pipelines. On the contrary, coal gasification has to be conducted near to the resource, even though it produces more expensive hydrogen-containing gas from a resource, which is less convenient for traditional applications and petrochemical production, than natural gas. In addition, everything depends on short-term prices, however, thorough preparation for the future requires longer-term decisions.

Intermediate energy-containing products and byproducts exist in HSC networks, which originate from many distributed districts. Biomass energy plants as well as some agricultural byproducts and waste appear seasonally, however, municipal biological wastes are constantly emitted.

Biomass is processed by gasification, while biological wastes produce biogas by anaerobic fermentation. The enrichment of the hydrogen content in the fermentation process is supported by some experimental results [8,9].

Energy-consuming, direct hydrogen-producing resources are electrical energy resulting from various chains, and hydrogen-containing gases that originate from SMR and gasification processes. The two corresponding kinds of hydrogen production processes are electrolysis and the different methods of gas separation for hydrogen enrichment, e.g. membrane separation, pressure swing adsorption, etc.

The produced raw hydrogen has to be conditioned in production sites by liquefaction or compression. The resultant liquidized or compressed hydrogen product needs a production or storage site for liquid or gaseous hydrogen, respectively.

Hydrogen can be transported from production to consumption sites by tanker trucks, tube trailers, railway tankers, railway tube cars or pipelines. Pure liquid or gaseous hydrogen needs to be stored at the site of consumption.

The utilization of hydrogen is connected to these storage sites. In addition to fueling cars and other vehicles, especially aircraft, hydrogen can be used to generate thermal energy as well as in various industrial processes, e.g. ammonia production, synthesis of organic products, metal processing, etc.

In the long-term development of energy supply, hydrogen can serve as a clean and safe energy carrier that contributes to the adoption of a low-carbon economy. Considering the slow but definite development towards a hydrogen economy, the optimal design, planning and operation of a Hydrogen Supply Chain have come to the fore of research over the last decade.

Supply chain network design is a dynamic field of research, not only in the context of HSC but in general. A comprehensive set of various fields of applications was reviewed by Govindan [10].

Regarding the generally applied modeling methods for the planning and design of HSC, a review from
2012 lists the main approaches [11] such as mathematical optimization as well as Geographic Information System (GIS) based models and frameworks. In conclusion, mainly mathematical programming-based optimization methods are used to design and plan HSCs worldwide.

In 2017, another paper [12] provided an overview once more about the actual situation on the basis of almost 100 references. Various mathematical optimization methods such as mixed-integer linear programming (MILP), mixed-integer nonlinear programming (MINLP), mathematical programming problem (MPP), mathematical optimization (MOP) as well as GIS-based approaches and transition models illustrate progress over the last five years. Regardless of some alternatively applied system dynamics (SD) and agent-based modeling (ABM) solutions, mathematical programming-based optimization methods are still the most widely used tools in planning and design.

This preferred approach is based on Mixed-Integer Linear Programming (MILP) in which binary variables denote the selection of technologies and establishment of transportation links, while continuous ones denote mass and energy flows, capacities of the supply chain nodes, as well as the cost and environmental performance metrics. These decision variables are optimized subject to mass balance constraints, capacity limitations and calculations of objective functions $[13,14]$.

Life Cycle Analysis (LCA) is an appropriate methodology to evaluate the environmental performance and potential impacts associated with a product system across its entire life cycle. Among various LCA studies that evaluated the environmental benefits of using hydrogen over conventional energy sources, the so-called well-towheels (WTW) studies - an LCA approach to evaluate the environmental advantages of alternative fuel vehicles (AFVs) over conventional engine vehicles across the entire automotive fuel pathway - are studied including hydrogen supply chains [15].

Multiple spatial and temporal scales imply a big challenge. Samsatli et al. [16] highlights the need for the combined consideration of various temporal scales. A simple example of this is that the representation of energy storage requires a short (e.g. hourly) timescale, so taking into consideration years-long planning may result in computationally intensive models. Dynamic simulation-based approaches (including the actually studied Programmable Process Structures) may contribute towards solutions to these problems.

Another big challenge is the question of managing uncertainty throughout the design optimization procedure.

Accounting for multiple criteria, e.g. costs, environmental impacts, safety, etc., is an obvious expectation of green supply chain design, while the incorporation of these issues generates another interesting task to be solved [17].

Many aspects of these challenges in design, deployment and operation in the field of HSC have been summarized in a recently published book [18]. 


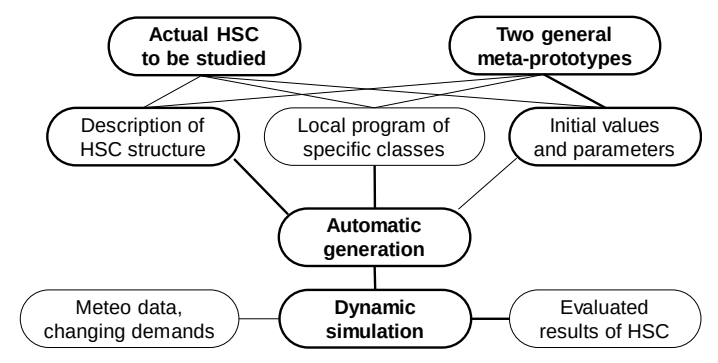

Figure 1: Scheme of HSC model generation by the method of Programmable Process Structures.

\section{Applied modeling method: Pro- grammable Process Structures}

By considering the aforementioned overview of challenges that computational model-supported HSC planning and operation face, a simple trial with our nonconventional approach was conducted. Accordingly, the methodology of Programmable Process Structures (PPS) $[19,20]$ was applied for the simulation-based analysis of a fictitious but typical Hydrogen Supply Chain.

In PPS (see Fig. 1), the locally programmable structure of process models can be generated from the GraphML-based graphical implementation of one state and one transition defining meta-prototypes, as well as from the unified textual description of the studied HSC network automatically resulting in a dynamic structure of the standardized state and transition elements. The programmable prototype elements that describe production, transformation, transportation, utilization and intermediate storage in the model can also be derived from the two general meta-prototypes. The freely editable, actual program of the prototypes contain symbolic input, parameter and output variables, as well as a locally executable GNU Prolog [21] code. In the various cases, the actual state and transition elements can be modeled with the same or a similar reusable local program, while the number of program prototypes is considerably less than the number of actual elements.

The state and transition elements of the Programmable HSC Structures can be parameterized and initialized concerning their case-specific prototypes. During the simulation, the actual elements are executed by the prototypes associated with them. This execution results in cyclically stepwise, connection-based communication of the state and transition elements of the programmed structure with the general-purpose kernel that works like an operational system.

When the simulation is run, the actual elements start with the initial conditions and parameters, while the output values are recalculated stepwise by taking into consideration the knowledge of input and parameter data in accordance with the associated local program prototypes. Distinguished input and output connectors as well as connections exist for the conservation measures (extensive/intensive properties) and signals, respectively. This solution supports the combined execution of the balance- based and signal-based functionalities, i.e. the hybrid models of hybrid processes.

\section{Modelling and analysis of Hydrogen Supply Chains by Programmable Pro- cess Structures}

\subsection{Description of the example HSC process network}

In the following sections, the following notations of Prolog syntax shall be used:

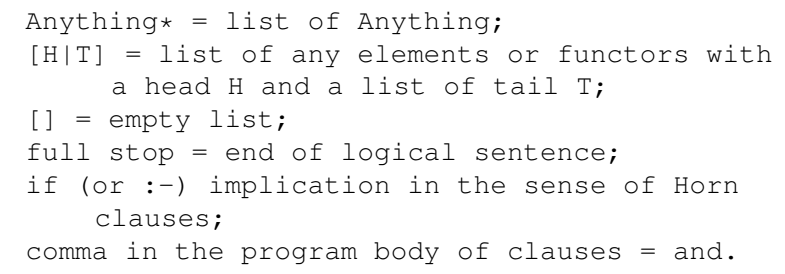

The general declarations of multi-compartment (multilevel, multi-scale) state and transition elements are as follows:

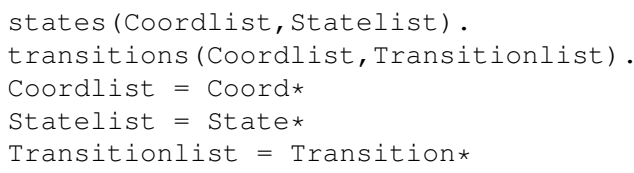

The transition-based declaration of the structures is described by the individual transitions as follows:

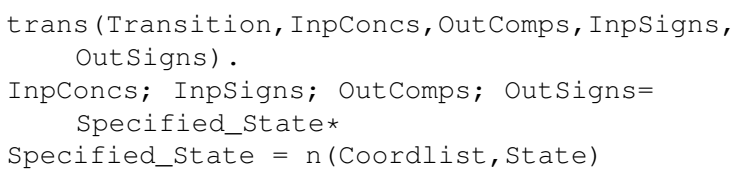

where the $\mathrm{n}($ ) functors of InpConcs, InpSigns, OutComps and OutSigns contain the coordinate and name of the state connected with the given input measure, input signal, output measure or output signal, respectively. This solution allows the same state names in the various compartments, levels or scales to be used because they are identified by the spatial coordinate and name together.

Our simplified example process network contains 38 state and 39 transition elements altogether in four regions, i.e. coordinates. The interpretation and abbreviation of the elements is explained in Table 1.

The declaration of the process network is illustrated by a characteristic part of the definitions as follows:

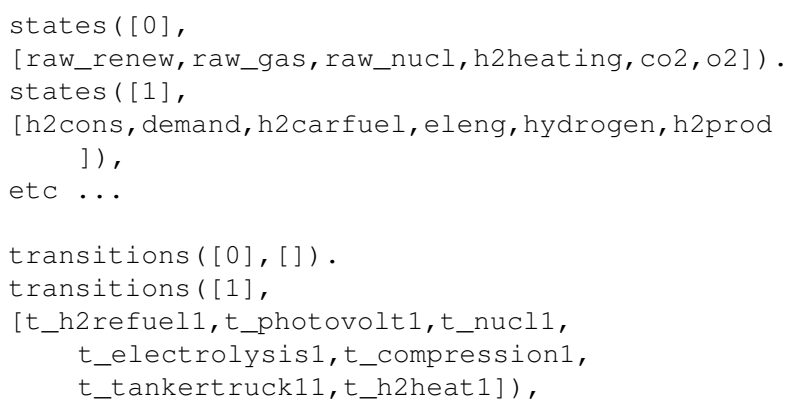


Table 1: List of involved state and transition elements.

\begin{tabular}{|c|c|c|}
\hline $\begin{array}{l}\text { Type of } \\
\text { element }\end{array}$ & Name of element & Short symbol \\
\hline \multirow{16}{*}{$\begin{array}{l}\text { State } \\
\text { element }\end{array}$} & Renewable resources & raw_renew \\
\hline & Cultivated land & raw_land \\
\hline & Natural gas & raw_gas \\
\hline & $\begin{array}{l}\text { Raw materials for nuclear en- } \\
\text { ergy }\end{array}$ & raw_nucl \\
\hline & Energy-producing biomass & biomass \\
\hline & Biological waste & biowaste \\
\hline & Electrical energy & eleng \\
\hline & Hydrogen-containing gas & h2contgas \\
\hline & Raw hydrogen produced & hydrogen \\
\hline & Compressed $\mathrm{H}_{2}$ produced & h2prod \\
\hline & $\begin{array}{l}\text { Compressed } \mathrm{H}_{2} \text { available for } \\
\text { consumers as a fuel }\end{array}$ & h2cons \\
\hline & Fuel demand of cars & demand \\
\hline & $\mathrm{H}_{2}$ utilized as a motor fuel & h2carfuel \\
\hline & Heat energy & h2heating \\
\hline & Carbon dioxide & $\mathrm{co} 2$ \\
\hline & Oxygen & $\circ 2$ \\
\hline \multirow{14}{*}{$\begin{array}{l}\text { Transition } \\
\text { element }\end{array}$} & Cultivation of land & t_cultivation \\
\hline & Photovoltaic process & t_photovolt \\
\hline & Wind power station & t_wind \\
\hline & Hydroelectric power station & t_hydro \\
\hline & Nuclear power station & t_nucl \\
\hline & Steam methane reforming & $t \_s m r$ \\
\hline & Biomass gasification & t_gasify \\
\hline & Anaerobic fermentation & t_ferment \\
\hline & Electrolysis & t_electrolysis \\
\hline & Gas separation & t_gassep \\
\hline & Compression & t_compression \\
\hline & Transportation (tanker truck) & $t \backslash$ tankertruck \\
\hline & Refueling cars (vehicles) & t_h2refuel \\
\hline & Heat production & t_h2heat \\
\hline
\end{tabular}

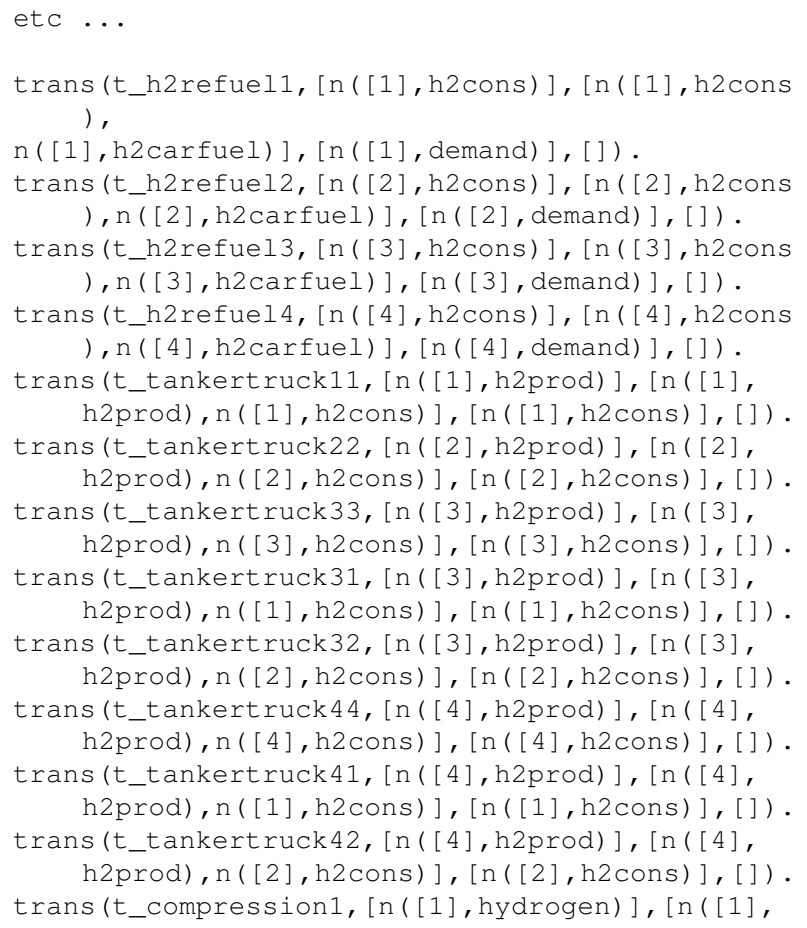

hydrogen), n([1], h2prod) ], [], [ ] ).

trans (t_compression2, [n ([2], hydrogen) ], [n ([2], hydrogen), n([2], h2prod) ], [], []).

trans (t_compression3, [n ([3], hydrogen) ], [n ([3], hydrogen), n([3], h2prod) ], [], [] ).

trans (t_compression4, [n ([4], hydrogen) ], [n ( [4], hydrogen), n([4], h2prod) ], [], [] ). etc ...

\subsection{Meta-prototypes-based generation and analysis of the example model}

Generation of the Programmable Process Structure originates from the GraphML description of general metaprototypes that belong to the methodology (see their illustration in the upper part of Fig. 2) and from the aforementioned description of the actual process network. The initial values and parameters of state elements as well as the parameters of transition elements can be added automatically from an Excel based text file. The generated general-purpose algorithm results in a GraphML file that contains the two meta-prototypes and structure of the whole HSC model in addition to the initial values and parameters.

The example structure is illustrated in Fig. 2. The GraphML model can be further edited by various graph editors (yEd Graph Editor to be precise). The structure also contains the two empty meta-prototypes.

The generated Programmable Process Structure describes all of the state (storage of resources, intermediates and products) and transition elements that represent the transformation and transportation in the various compartments (spatial scales) individually, because they may have different parameters and connections. By default, the transportations belong to the sending compartment. This representation does not result in the multiplication of the program code because many elements are executed by the same meta-prototypes that contain the local programs (see the next Section).

The connections from the state to transition elements represent the causally right transfer of quantitative and qualitative amounts and signals necessary for the calculation of the transitions. The connections from the transition to state elements represent the increases and decreases in the corresponding additive measures as well as the calculated signals. Accordingly, the model is prepared for the common but distinguished representation of the extensive/intensive properties as well as of the signals and rules.

Consequently, the Programmable Process Structures make both the elements and structure visible during the simulation process. This supports the easy (optionally multiscale) temporal consideration of external data, e.g. related to meteorology, the climate and supply \& demand. Moreover, the flux routes and influence routes can be seen and evaluated explicitly.

Furthermore, this (apparently too abundant) knowledge representation makes the unified execution of quite 


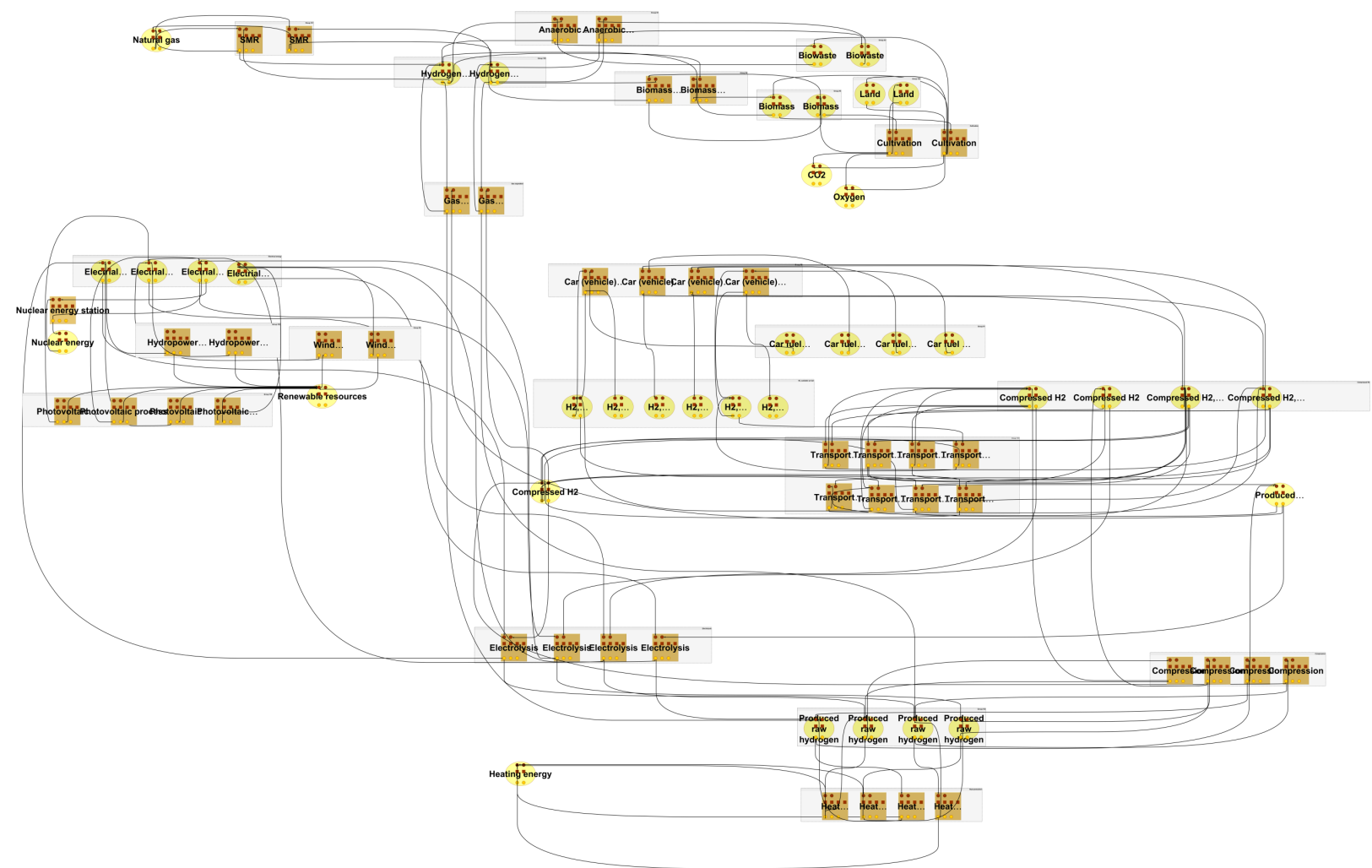

Figure 2: Programmable Process Structure of the investigated HSC.

different hybrid dynamical models possible by applying the same kernel program.

\section{Meta-prototypes}
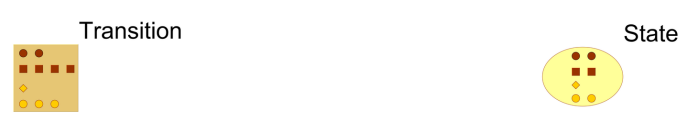

\section{Actual, case specific} prototypes

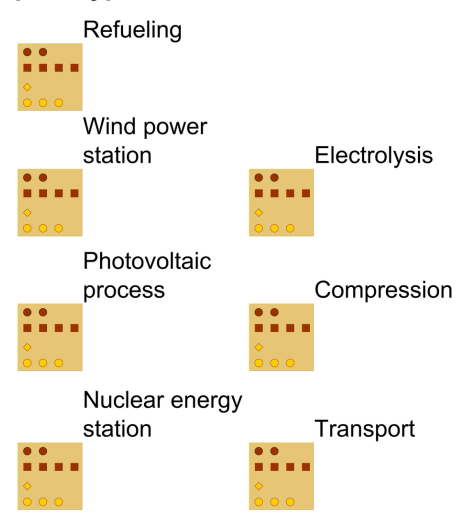

Figure 3: Some meta-prototypes-based case-specific prototypes of HSC networks.

\subsection{Meta-prototypes-based functional proto- types of the example model}

First, the programmable prototypes have to be prepared by making copies from the application-independent meta-prototypes. Afterwards, the declaration of the actual local program follows. The implementation of state and transition prototype elements of the HSC model needs creative collaboration between the modeller and field experts. The preparation of the case-specific local programs is supported by the meta-knowledge embedded in the meta-prototypes, in accordance with the generated Programmable Process Structure. The basic meta-prototypes and some actual programmed prototypes are illustrated in Fig. 3.

The example prototypes in Fig. 3 are as follows:

\section{Transition prototypes:}

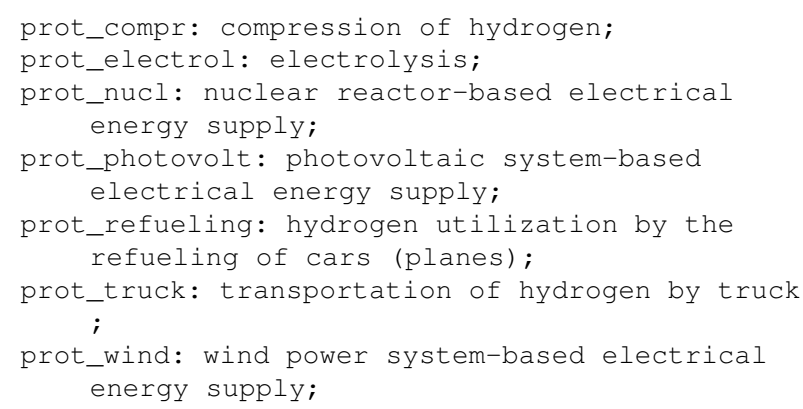

\section{State prototypes:}

prot_demand: scheduled hydrogen demand; 
prot_storage: hydrogen storage;

prot_condstorage: hydrogen storage, associated with condition signaling capabilities.

As an example, see the simplified local program of prot_photovolt that calculates the produced electrical energy as a function of the actual meteorological conditions. This textual code is to be embedded into the respective prototype element as follows:

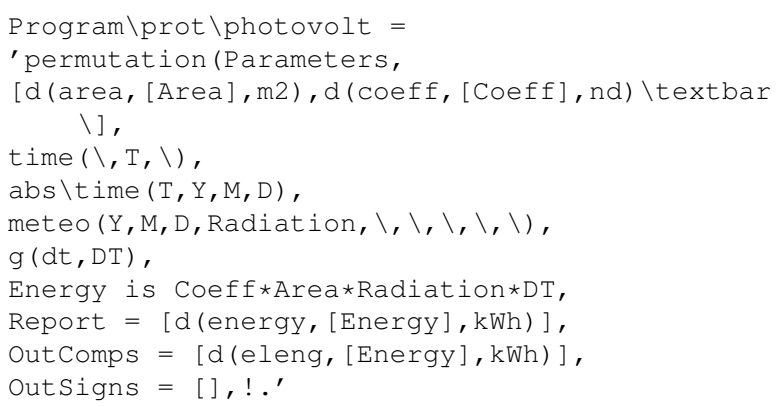

\subsection{Generation and simulation of the example HSC system}

Programming of the process structure is followed by its automatic interpretation into the executable dynamic databases of the given model. Now the general model generator of the kernel reads the GraphML file and generates:

- the facts that describe the actual state $\&$ transition elements and those that determine state $\rightarrow$ transition and transition $\rightarrow$ state connections in the "user" file;

- the clauses that declare the program prototypes in the "expert" file.

The resultant user and expert files contain a detailed description of the process model, including the locally executable program prototypes as well as the actual elements and connections that contain the initial data and parameters.

Having generated the model, the executable code has to be supplemented by the (optionally scale-specific) global data, e.g. $g(d t, D T)$, that also declares the main time step in the previous example code.

Dynamic simulation is executed by the general kernel program that comprises the cyclically repeated processing of state elements, state $\rightarrow$ transition connections, transition elements and transition $\rightarrow$ state connections.

For example, the execution of all transitions associated with the prototype of prot_photovolt are executed by the the unification of them, e.g.

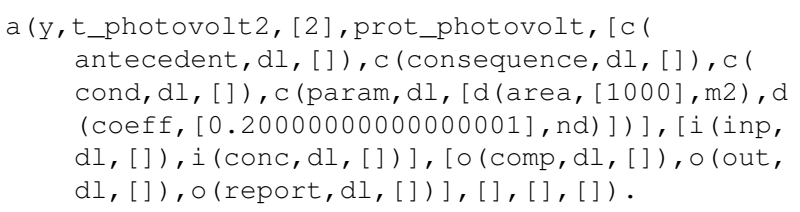

is unified with the prototype

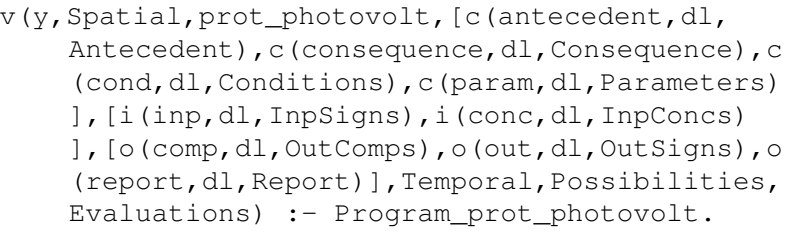

where Program_prot_photovolt refers to the example code above.

The variables in prototypes are described by alphanumeric symbols that start with a capital letter. The variables and constants in the functors $i()$, $c()$ and $\circ()$ are described by lists of the triplets d(Identifier, Variable_list, Instructions).

\subsection{Analysis of the simulated results}

Some characteristic parameters in these illustrative examples were the following:

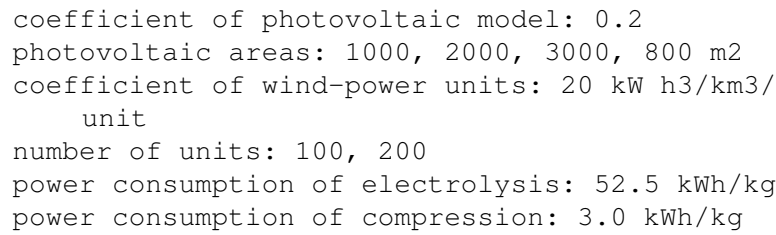

Some of the simulated results are illustrated with a specific part of a simplified HSC model. In this case, a seasonally and randomly changing continuous increase in the demand of cars for hydrogen fueling over the next decade was assumed, as can be seen in Fig. 4.

In terms of production, the available capacity of photovoltaic power stations and wind farms in the studied region was investigated. These renewable resources produce electrical energy depending on the actual meteorological conditions (solar radiation and wind speed). However, the available climate scenarios did not contain data concerning radiation and wind speed, so meteorological data from the past ten years was used to imitate realistic changes in radiation and wind speed. The calculated electrical energy production of the investigated system is illustrated in Fig. 5.

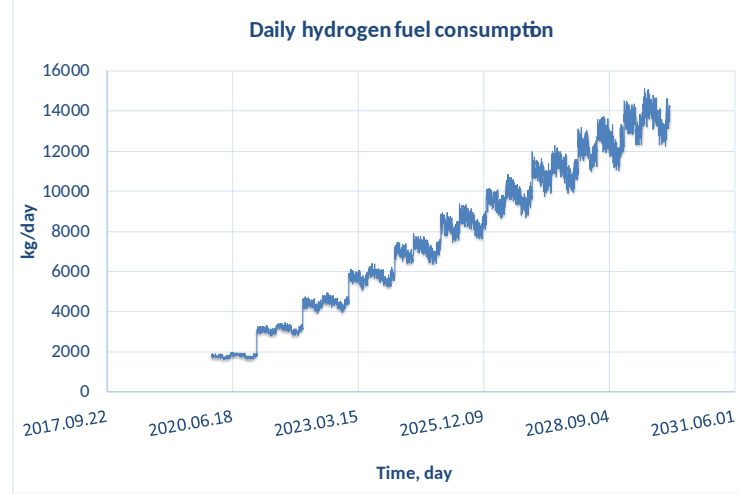

Figure 4: Hypothetical data for the seasonally changing and increasing demand for hydrogen. 


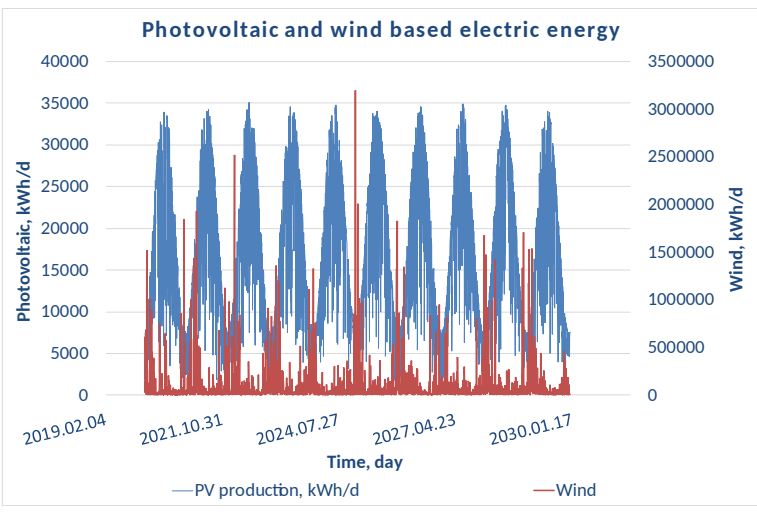

Figure 5: Electrical energy production of the involved wind power and photovoltaic systems (to be converted into hydrogen by electrolysis).

In the studied area, wind power can produce more electrical energy than photovoltaic systems, however, it fluctuates very erratically. The contribution of photovoltaic systems is smoother with characteristic seasonal changes.

Increasing demands for hydrogen production can be met by more renewable energy production or additional hydrogen production from the available electrical energy (e.g. originating from nuclear power stations) and additional hydrogen production (e.g. from Steam-Methane Reforming, biomass gasification, etc.).

In the case of lower fuel demands for cars (over a short initial period), the solar energy-based renewable resources can produce the necessary energy for hydrogen production. Furthermore, some extraordinary surplus amounts can be produced over randomly appearing short periods by wind farms, as can be seen in Figs. 5 and 6 .

The results underline that dynamic simulation models need to be applied to solve planning problems in the case of rapidly changing supply and demand.

\section{Discussion}

As has been summarized in the Introduction, mainstream HSC planning and operation applies mathematical programming, where a simplified model is usually embedded in a superstructure-based optionally multi-objective optimization program.

The suggested non-conventional approach might have some potential advantages. Having analyzed the characteristics of Hydrogen Supply Chains and their typical elements, Programmable Process Structures may offer some useful options:

- the methodology tolerates case-specific multiscale extensions (e.g. when a proportion of the elements is in need of a more detailed model and/or refined spatial or temporal resolution).

- by taking into consideration the intensive development of new hydrogen-producing methods, Pro-

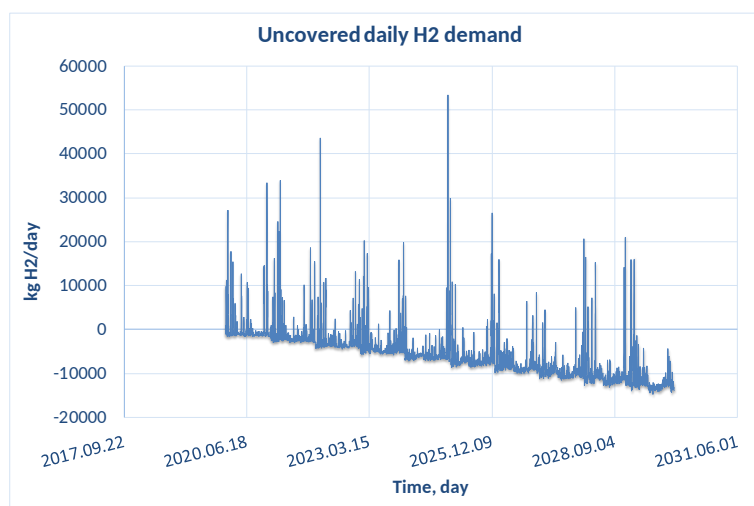

Figure 6: The change in hydrogen demand produced by the available direct renewable resources.

grammable Process Structures are capable of embedding any detailed prototypes of nonlinear dynamic models into existing structures.

- the extensibility of Programmable Process Structures favours stepwise structural and functional changes in supply and demand without reformulation of the model.

- optional multiscale compartmentalization helps to represent multiple districts and sites via the optional connection between spatial coordinates of the elements with an external GIS tool.

- individual elements and connections can be associated with a time-driven execution at given points in time and/or over prescribed time intervals.

- material and energy balances can be combined with signal and rule-based, event-driven processes.

- a Programmable Process Structure has built-in capabilities for robust communication with external (e.g. meteorological) databases and collaborating (e.g. metaheuristic optimization) programs.

- the method supports the handling of the extraordinary uncertainty of coefficients of cost and some other parameters over a longer time horizon. The embedded elementary evaluations and elements of evaluation may be prepared for a multi-objective case involving a set of time-invariant natural objective functions.

\section{Conclusion}

Regardless of the well-established mathematical programming-based methods of the planning and operation of Hydrogen Supply Chains, problem-solving in terms of these systems might also require an easily modifiable, generic dynamic simulation-based approach.

As was also shown by the studied illustrative example, the representation of energy storage corresponding to renewable resources requires a short (e.g. hourly, daily) 
timescale as opposed to a years-long planning time horizon.

The reason for this is that these large-scale, long-term supply chains are not only affected by uncertainly changing demands, but by erratically changing supply, partly originating from natural resources, determined by erratically changing extreme weather conditions and a slowly changing climate.

By considering these challenges with regard to the planning and operation of a Hydrogen Supply Chain, the non-conventional modeling and simulation methodology of Programmable Process Structures was implemented as developed in other multidisciplinary fields.

The suggested Programmable Process Structures may contribute to the solution to these problems. Programmable Process Structures of HSC models can be generated from the description of a (optionally geographically determined and multiscale) process network and two general functional meta-prototypes, automatically. The case-specific, functional program prototypes can be derived from the two meta-prototypes. Simultaneously, the actual elements of the process network can be parameterized and initialized according to the case-specific prototypes. The simulation of the model can be solved by a general-purpose kernel program. The method also allows different time steps for the various classes of elements to be used.

The application of Programmable Process Structures was illustrated by a fictitious example of a simple Hydrogen Supply Chain.

Planned future work, in collaboration with HSC experts, will focus on the application of a repeated forward/backward simulation-based iterative methodology to reach a reasonable compromise between changing demands and supplies. Furthermore, Programmable Process Structures will be combined with metaheuristic optimizers for the multi-objective optimization of HSC planning.

\section{Notations}

$\begin{array}{ll}\begin{array}{l}\text { GIS } \\ \text { GraphML }\end{array} & \begin{array}{l}\text { Geographic Information System } \\ \text { A comprehensive and easy-to-use file } \\ \text { format for graphs }\end{array} \\ \text { HSC } & \text { Hydrogen Supply Chain } \\ \text { MILP } & \text { Mixed Integer Linear Programming } \\ \text { MINLP } & \text { Mixed-Integer Nonlinear Programming } \\ \text { MPP } & \text { Multi-period problems } \\ \text { MOP } & \text { Multi-objective problems } \\ \text { PPS } & \text { Programmable Process Structure }\end{array}$

\section{Acknowledgement}

This research was supported by the TÉT_16-1-20160116 French-Hungarian bilateral project.

\section{REFERENCES}

[1] Hydrogen Deployment. Special Section of Chemical Engineering Progress, Journal of the Ameri- can Institute of Chemical Engineers, August 2019, aiche.org/cep

[2] Calloway, B.; McWhorter, S.; James, W.: Advancements in hydrogen deployment. In: Special Section of CEP Magazine, August 2019, https://www. aiche.org/resources/publications/cep/2019/ august/advancements-hydrogen-deployment

[3] De-Leon Almaraz, S.: Multi-objective optimisation of a Hydrogen Supply Chain. Ph.D. Thesis, 2014, Université de Toulouse.

[4] De-Leon Almaraz, S.; Azzaro-Pantel, C.: Chapter 4 - Design and Optimization of Hydrogen Supply Chains for a Sustainable Future. In: Hydrogen Economy, Eds. Scipioni, A., Manzardo, A., Ren, J., (Academic Press, 2017), pp. 85-120 DOI: 10.1016/B978-0-12-811132-1.00004-3

[5] Varga, M.; Csukás, B.: Meta-modeling of Hydrogen Supply Chains: a Programmable Structure based representation. In: (Azzaro-Pantel, C. ed.) Hydrogen Supply Chain: Design, Deployment and Operation, (Elsevier, 2018) DOI: 10.1016/B978-0-12-811197$0.00014-2$

[6] Show, K.Y.; Yan, Y.; Zong, C.; Guo, N.; Chang, J.S.; Lee, D.J.: State of the art and challenges of biohydrogen from microalgae, Bioresource Technol., 2019, 289, 121747 DOI: 10.1016/j.biortech.2019.121747.

[7] Sivaram, V.: Taming the Sun: Innovations to Harness Solar Energy and Power the Planet, (MIT Press, 2018), pp. 392 ISBN-13: 978-0262037686

[8] Wang, J.; Yin, Y.: Principle and application of different pretreatment methods for enriching hydrogen-producing bacteria from mixed cultures, Int. J. Hydrogen Energ., 2017, 42(8), 4804-4823 DOI: 10.1016/j.ijhydene.2017.01.135.

[9] Bakonyi, P.; Kumar, G.; Koók, L.; Tóth, G.; Rózsenberszki, T.; Bélafi-Bakó, K.; Nemestóthy, N.: Microbial electrohydrogenesis linked to dark fermentation as integrated application for enhanced biohydrogen production: A review on process characteristics, experiences and lessons, Bioresource Technol., 2018, 251, 381-389 DOI: 10.1016/j.biortech.2017.12.064.

[10] Govindan, K.; Fattahi, M.; Keyvanshokooh, E.: Supply chain network design under uncertainty: A comprehensive review and future research directions, Eur. J. Oper. Res., 2017, 263, 108-141 DOI: 10.1016/j.ejor.2017.04.009

[11] Dagdougui, H.: Models, methods and approaches for the planning and design of the future hydrogen supply chain, Int. J. Hydrogen Energy, 2012, 37, 5318-5327 DOI: 10.1016/j.ijhydene.2011.08.041

[12] Maryam, S.: Review of modelling approaches used in the HSC context for the UK, Int. J. Hydrogen Energy, 2017, 42(39), 24927-24938 DOI: 10.1016/j.ijhydene.2017.04.303

[13] Grossmann, I.E.; Guillen-Gosalbez, G.: Scope for the application of mathematical programming techniques in the synthesis and planning of sustainable 
processes, Comput. Chem. Eng., 2010, 34(9), 13651376 DOI: 10.1016/j.compchemeng.2009.11.012

[14] Guillen-Gosalbez, G.; Mele, F.D.; Grossmann, I.E.: A bi-criterion optimization approach for the design and planning of hydrogen supply chains for vehicle use; AIChE J., 2010, 56(3), 650-667 DOI: 10.1002/aic. 12024

[15] Kudoh, Y; Ozawa, A: Life Cycle Assessment of Hydrogen Supply Chain: A Case Study for Japanese Automotive Use. In: Azzaro-Pantel, C.: Hydrogen Supply Chains - Design, Deployment and Operation (Academic Press, Elsevier, 2018), Chapter 15, pp. 499 DOI: 10.1016/B978-0-12-811197-0.09988-7

[16] Samsatli, S.; Staffell, I.; Samsatli, N.J.: Optimal design and operation of integrated windhydrogen-electricity networks for decarbonising the domestic transport sector in Great Britain, Int. J. Hydrogen Energy, 2016, 41, 447-475 DOI: 10.1016/j.ijhydene.2015.10.032

[17] Nurjanni, K.P.; Carvalho, M.S.; Costa, L.: Green supply chain design: A mathematical modeling approach based on a multi-objective optimization model, Int. J. Prod. Econ., 2017, 183, 421-432 DOI: 10.1016/j.ijpe.2016.08.028

[18] Azzaro-Pantel, C.: Hydrogen Supply Chains - Design, Deployment and Operation (Academic Press, Elsevier, 2018), pp. 588 DOI: 10.1016/B978-0-12-8111970.09988-7

[19] Varga, M.; Csukás, B.: Programmable Process Structures, Generated from a Network and from Functional Meta-Prototypes. In: 2017 AIChE Annual Meeting Proceedings, Computing and Systems Technology Division, Software Tools and Implementations for Process Systems Engineering. Minneapolis (MN), USA, 2017. Paper 448e https://www.aiche.org

[20] Varga, M.; Prokop, A.; Csukás, B.: Biosystem models, generated from a complex rule/reaction/influence network and from two functionality prototypes, BioSystems, 2017, 152, 24-43 DOI: 10.1016/j.biosystems.2016.12.005

[21] http://www.gprolog.org/ 